\title{
Testing for ARCH in the Presence of Additive Outliers
}

\author{
Dick van Dijk* \\ Tinbergen Institute, Erasmus University Rotterdam \\ Philip Hans Franses \\ Rotterdam Institute for Business Economic Studies and \\ Econometric Institute, Erasmus University Rotterdam \\ André Lucas \\ Financial Sector Management, Free University Amsterdam
}

October 14, 1996

\begin{abstract}
In this paper we investigate the properties of the Lagrange Multiplier (LM) test for autoregressive conditional heteroskedasticity (ARCH) and generalized ARCH (GARCH) in the presence of additive outliers (AO's). We show analytically that both the asymptotic size and power are adversely affected if AO's are neglected: the test rejects the null hypothesis of homoskedasticity too often when it is in fact true, while the test has difficulty detecting genuine GARCH effects. Several Monte Carlo experiments show that these phenomena occur in small samples as well. We design and implement a robust test, which has better size and power properties than the conventional test in the presence of AO's. Applications to the French industrial production series and weekly returns of the Spanish peseta/US dollar exchange rate reveal that, sometimes, apparent GARCH effects may be due to only a small number of outliers and, conversely, that genuine GARCH effects can be masked by outliers.

Keywords: Generalized autoregressive conditional heteroskedasticity, Lagrange Multiplier test, Outliers, Robust testing.
\end{abstract}

JEL classification: $\mathrm{C} 12, \mathrm{C} 22$

${ }^{*}$ Correspondence to Dick van Dijk, Tinbergen Institute, Burg. Oudlaan 50, NL-3062 PA Rotterdam, The Netherlands, Email: dvandijk@tir.few.eur.nl 


\section{Introduction}

A common feature of many financial time series, such as returns on exchange rates and stock market indices, is that irregular data points tend to cluster. Large (small) absolute realizations typically are followed by large (small) realizations of either sign. Therefore, such clusters can be viewed as corresponding to periods with high (low) volatility. Given that volatility, as a measure of risk, is a dominant element in many financial analyses, it seems important to try to capture the behavior of volatility in an econometric time series model. The autoregressive conditional heteroskedasticity (GARCH) and generalized ARCH (GARCH) models, introduced by Engle (1982) and Bollerslev (1986), respectively, are by now the most widely used models for this purpose, see Bollerslev et al. (1992), Bera and Higgins (1993), and Bollerslev et al. (1994) for recent surveys.

The GARCH model can describe the excess kurtosis and the positive and slowly decaying autocorrelations in the squared observations, which are characteristic properties of many financial time series. However, Teräsvirta (1996) shows that the GARCH model with normal conditional errors cannot capture these stylized facts completely. In particular, the excess kurtosis cannot be completely accomodated. Teräsvirta suggests that models with fat-tailed conditional distributions, such as the Student $t$, might do better in this respect. When such GARCH- $t$ models are applied in practice, it often appears that the estimated residuals still have high excess kurtosis, see, e.g., Baillie and Bollerslev (1989). Hence, there may remain observations that cannot be described satisfactorily by an extended GARCH model. Such data points may correspond to another data generating process (DGP), possibly with markedly distinct distributional properties from the DGP of the other observations. A typical example of an aberrant observation is an additive outlier (AO), see, e.g., Martin and Yohai (1986) for a definition. Neglecting AO's in an observed time series can suggest leptokurtic behavior. In particular, a short sequence of two AO's already 
results in a positive bias in the first order autocorrelation of the squared observations. Such a sequence of AO's may, for example, be caused by an overreaction to some news fact, while this reaction is corrected in a subsequent period. On the other hand, low order autocorrelation coefficients are biased towards zero by isolated AO's, and, hence, neglecting AO's may blur inference on conditional heteroskedasticity. In sum, it seems worthwhile to account for the presence of both GARCH effects and outliers when dealing with financial time series. Moreover, as the economic interpretation of both phenomena is rather different, statistical procedures are called for that separate the effects of outliers from those of GARCH behavior.

In this paper, we focus on the first step in modeling financial time series, by considering the sensitivity of tests for conditional heteroskedasticity to the presence of AO's. We focus on the Lagrange Multiplier (LM) test put forward in Engle (1982), as this test is by far the most popular method to test for ARCH. An additional motivation for considering the effect of AO's on this ARCH test is given by the observation that it is applied routinely to the residuals of a fitted econometric time series model. In fact, many computer packages contain this test as a diagnostic check. Therefore, it seems warranted to examine the properties of this test while allowing for the presence of AO's. We show analytically that both the size and power of the LM test are adversely affected by neglected AO's and construct a robust test that can handle AO's much better.

The outline of this paper is as follows. In section 2, the GARCH model and the associated standard LM test are briefly discussed. Section 3 formally investigates the effects of additive outliers on the asymptotic distribution of this standard test statistic. An outlier robust variant of the LM test is developed in section 4 . In section 5, Monte Carlo experiments are used to investigate the small sample properties of both the conventional and the robust test. The robust test is shown to perform much better in terms of size and power. Empirical illustrations are given in section 6, where the LM tests are applied to the French industrial production series and to 
weekly returns of the Spanish peseta/US dollar exchange rate. Section 7 concludes with suggestions for further research.

\section{The LM test for GARCH}

Consider the autoregressive (AR) model of order $m$ with $\operatorname{GARCH}(p, q)$ disturbances for a univariate time series $y_{t}$ with mean $\mu$,

$$
\begin{aligned}
\phi(L)\left(y_{t}-\mu\right) & =\varepsilon_{t}, t=1, \ldots, T \\
\varepsilon_{t} \mid \Omega_{t-1} & \sim\left(0, h_{t}\right), \\
h_{t} & =\omega+\sum_{i=1}^{q} \alpha_{i} \varepsilon_{t-i}^{2}+\sum_{j=1}^{p} \beta_{j} h_{t-j} \equiv \omega+\alpha(L) \varepsilon_{t-1}^{2}+\beta(L) h_{t-1}^{2},
\end{aligned}
$$

where $\Omega_{t-1}$ represents the information set available at time $t-1$ and $\phi(L)$ is a polynomial of order $m$ in the lag operator $L$, defined by $L^{k} y_{t}=y_{t-k}$. We assume that the roots of $\phi(z)$ lie outside the unit circle. For the conditional variance of $\varepsilon_{t}$ to be strictly positive, the parameters in (3) have to satisfy $\omega>0, \alpha_{i} \geq 0, i=1, \ldots, q$, and $\beta_{j} \geq 0, j=1 \ldots, p$. A necessary and sufficient condition for the existence of the unconditional variance of $\varepsilon_{t}$ is given by $\sum_{i=1}^{q} \alpha_{i}+\sum_{j=1}^{p} \beta_{j}<1$, see Bollerslev (1986). When this condition is satisfied, this unconditional variance is given by $E\left(\varepsilon_{t}^{2}\right)=\omega /\left(1-\sum_{i=1}^{q} \alpha_{i}-\sum_{j=1}^{p} \beta_{j}\right)$. The conditional distribution of the disturbances $\varepsilon_{t}$ usually is assumed to be either normal or Student $t$. If $p=0$, the error process reduces to the $\operatorname{ARCH}(q)$ process, see Engle (1982), while for $p=q=0$, $\varepsilon_{t}$ is simply white noise.

Although nowadays estimation of GARCH models has become fairly straightforward, it still is sound practice to start any specification analysis with testing for the presence of ARCH effects. The LM test procedure has become one of the most popular methods to test white noise errors against the alternative of conditional heteroskedasticity, as it only requires estimation of the model under the null hypothesis. The testing problem can be formulated as testing the null hypothesis $H_{0}: \alpha_{1}=\ldots=\alpha_{q}=\beta_{1}=\ldots=\beta_{p}=0$, against the alternative $H_{a}: \alpha_{i}>0, \beta_{j}>0$ 
for at least one $i=1, \ldots, q$ and $j=1, \ldots, p$. Lee (1991) shows that the LM test against this $\operatorname{GARCH}(p, q)$ alternative is the same as the LM test against the alternative of $\operatorname{ARCH}(q)$ errors. This follows from the fact that under the null hypothesis, the gradient of the log likelihood of the $\operatorname{AR}(m)-\operatorname{GARCH}(p, q)$ model (1)-(3) with respect to the parameters $\beta_{1}, \ldots, \beta_{p}$ is equal to zero. The $\operatorname{LM}$ test against $\operatorname{ARCH}(q)$ errors was developed by Engle (1982) and is given by

$$
\xi^{*}=\frac{1}{2} \hat{f}^{\prime} \hat{Z}\left(\hat{Z}^{\prime} \hat{Z}\right)^{-1} \hat{Z}^{\prime} \hat{f}
$$

where $\hat{Z}^{\prime}=\left(\hat{z}_{1}^{\prime}, \ldots, \hat{z}_{T}^{\prime}\right), \hat{z}_{t}=\left(1, \hat{\varepsilon}_{t-1}^{2}, \ldots, \hat{\varepsilon}_{t-q}^{2}\right)^{\prime}$, and $\hat{f}^{\prime}=\left(\hat{f}_{1}^{\prime}, \ldots, \hat{f}_{T}^{\prime}\right), \hat{f}_{t}=\left(\hat{\varepsilon}_{t}^{2} / \hat{\sigma}^{2}-\right.$ 1), with $\hat{\sigma}^{2}=\sum_{t=1}^{T} \hat{\varepsilon}_{t}^{2} / T$. In (4), least squares estimates of the conditional mean equation (1), obtained under the null of no $\mathrm{ARCH}$, are used. This statistic takes the same form as the well-known heteroskedasticity test of Breusch and Pagan (1979). Engle (1982) notes that the test does not depend on the linear form of the conditional variance function $h_{t}$ in (3), which implies that the same test statistic results for any specification of $h_{t}$ which depends only on the past squared disturbances $\varepsilon_{t-i}^{2}, \ldots, \varepsilon_{t-q}^{2}$.

Under the assumption of conditional normality of the $\varepsilon_{t}$ 's, an asymptotically equivalent statistic is given by

$$
\xi=\frac{T \hat{f}^{\prime} \hat{Z}\left(\hat{Z}^{\prime} \hat{Z}\right)^{-1} \hat{Z}^{\prime} \hat{f}}{\hat{f}^{\prime} \hat{f}}=T R^{2},
$$

where $R^{2}$ is the uncentered coefficient of determination of an auxiliary regression of the squared LS residuals $\hat{\varepsilon}_{t}^{2}$ on an intercept and $q$ lags $\hat{\varepsilon}_{t-1}^{2}$ through $\hat{\varepsilon}_{t-q}^{2}$. Under the null hypothesis of no $(\mathrm{G}) \mathrm{ARCH}$, the LM statistic $\xi$ is asymptotically $\chi^{2}$ distributed with $q$ degrees of freedom. Weiss (1986) shows that, subject to certain moment conditions, this LM test is also appropriate for nonnormal conditional distributions, see also Koenker (1981).

The small sample properties of the LM test $\xi$ have been investigated by Diebold and Pauly (1989), Gregory (1989), and Lee and King (1993), among others. The main findings of these studies can be summarized as follows. One consistently finds 
that the actual size of the test is lower than its nominal size, while the power is reasonable, although not overwhelming. Diebold and Pauly (1989) report that the power of the exact test (4) seems better than the power of its asymptotic equivalent (5). However, for the test against an $\operatorname{ARCH}(1)$ or $\operatorname{GARCH}(1,1)$ alternative, the differences disappear already for sample sizes of 100 observations. Gregory (1989) finds that the power of the LM test is sensitive to departures from symmetry in the conditional error distribution, while both Gregory (1989) and Lee and King (1993) show that the LM statistic is fairly robust against leptokurtic conditional error distributions.

In this paper, we are concerned with the potential effects on the LM test for $\mathrm{ARCH}$ of a particular deviation from conditional normality, namely the presence of additive outliers. In the next section, we focus on these effects in more detail.

\section{Additive outliers, size, and power}

In this section we demonstrate the effects of AO's on the asymptotic distribution of the LM test for ARCH. In subsection 3.1 we present the effect of isolated additive outliers on the level of the ARCH test if the model in levels contains an AR specification, which is of importance for the use of the LM test as a diagnostic check. In subsection 3.2 we derive the effect of patchy additive outliers on the level of the test. Finally, in subsection 3.3 we obtain the effect of isolated additive outliers on the power of the ARCH test, which seems relevant for the application of the test to financial time series.

In order to simplify the exposition and to abstract from unnecessary complications, we focus on the $\mathrm{AR}(1)$ model with zero mean and $\mathrm{ARCH}(1)$ errors,

$$
\begin{aligned}
y_{t} & =\phi y_{t-1}+\varepsilon_{t}, \\
\varepsilon_{t} \mid \Omega_{t-1} & \sim I N\left(0, h_{t}\right), \\
h_{t} & =\omega+\alpha \varepsilon_{t-1}^{2},
\end{aligned}
$$


with $\omega>0,0 \leq \alpha<1$ and $-1<\phi<1$. The series $y_{t}$ is observed with error as

$$
x_{t}=y_{t}+\zeta \delta_{t}
$$

where $\left\{\delta_{t}\right\}$ is a stochastic contamination process, which takes nonzero values with positive probability, and where $\zeta>0$ is a constant indicating the magnitude of the outliers.

Deriving the effect of outliers on the asymptotic distribution of the ARCH test $\xi$ in (5) is nontrivial, because 8-th order (cross-)moments of the different stochastic variables in (6) through (9) are involved. Therefore, instead of deriving the exact effects, we follow a slightly different route. In order to avoid presenting unduly lengthy derivations, we concentrate on the effect of outliers on the noncentrality parameter of the asymptotic $\chi^{2}$ distribution of the ARCH test, compare Koenker (1981). To be more precise, we only look at the effect of outliers on the main determinant of this noncentrality parameter, namely the expectation of

$$
\left(\frac{\left(x_{t}-\tilde{\phi} x_{t-1}\right)^{2}}{\tilde{\sigma}^{2}}-1\right)\left(x_{t-1}-\tilde{\phi} x_{t-2}\right)^{2},
$$

where $\tilde{\phi}$ is the probability limit of the ordinary least squares (OLS) estimator of the $\operatorname{AR}(1)$ parameter, and where $\tilde{\sigma}^{2}$ is the probability limit of the OLS estimator of the variance of the regression errors. The noncentrality parameter is given by the squared expectation of (10) divided by the variance of (10). For simplicity, we abstract from the effect of outliers on the variance of (10). We also abstract from the fact that in practice the test is based on a series of finite length. The finite sample bias is of course interesting on its own, but its effect on the noncentrality parameter is of a lower order than the expectation of (10). By focusing on the expectation of (10) only, we obtain tractable results that reveal the main consequences of outliers on the standard ARCH test. These consequences are further illustrated by means of Monte Carlo simulations in section 5. 


\subsection{A homoskedastic $\mathrm{AR}(1)$ and isolated additive outliers}

We first consider an $\operatorname{AR}(1)$ model with homoskedastic errors $\varepsilon_{t}$ and isolated additive outliers, so $\alpha=0$ in (8) and $\delta_{t}$ is an i.i.d. process, with $P\left(\delta_{t}=0\right)=1-\pi$, $P\left(\delta_{t}=1\right)=P\left(\delta_{t}=-1\right)=\pi / 2$. In this situation, additive outliers have several effects, see, e.g., Denby and Martin (1979), Bustos and Yohai (1986), and Martin and Yohai (1986). First, they cause a bias in the estimate of the autoregressive parameter $\phi$. Second, additive outliers affect the estimate of the error variance. Both effects have implications for the noncentrality parameter of the ARCH test.

The probability limit of the OLS estimate of the AR(1) parameter $\phi$ under isolated $\mathrm{AO}$ contamination is given by

$$
\operatorname{plim}_{T \rightarrow \infty} \hat{\phi} \equiv \tilde{\phi}=\frac{E\left(x_{t} x_{t-1}\right)}{E\left(x_{t-1}^{2}\right)}=\frac{\phi}{1+\pi \zeta^{2}\left(1-\phi^{2}\right) / \omega} .
$$

The OLS residuals are given by

$$
\begin{aligned}
\hat{\varepsilon}_{t} & =x_{t}-\tilde{\phi} x_{t-1} \\
& =\varepsilon_{t}+\zeta \delta_{t}-\tilde{\phi} \zeta \delta_{t-1}+(\phi-\tilde{\phi}) y_{t-1},
\end{aligned}
$$

from which the estimated error variance follows easily as being

$$
\begin{aligned}
\tilde{\sigma}^{2} & =E\left(\hat{\varepsilon}_{t}^{2}\right) \\
& =\omega\left(1+\frac{(\phi-\tilde{\phi})^{2}}{1-\phi^{2}}\right)+\pi \zeta^{2}\left(1+\tilde{\phi}^{2}\right) .
\end{aligned}
$$

The derivation of the exact expectation of (10) is much more cumbersome. Using computer algebra we obtain the correct expression, which is available from the corresponding author. To save space, we only present figures of the expectation of (10) for several parameter configurations in Figure 1.

\section{- insert Figure 1 -}

The first thing to notice from this Figure is the increase in the expectation for larger values of $\zeta$. This follows directly from the fact that a larger $\zeta$ causes a larger 
bias in the autoregressive parameter estimate. Also, the increasing behavior in $\phi$ is evident; if $\phi$ is larger, the discrepancy between the true residuals $\varepsilon_{t}$ and the contaminated residuals $\hat{\varepsilon}_{t}$ becomes larger if outliers are added. This again follows from the bias in the estimator for $\phi$, given in (11). Finally, if $\pi$ increases, the expectation decreases and, hence, the value of the noncentrality parameter decreases towards zero. In other words, the distribution gets closer to the nominal distribution if the fraction of contamination becomes larger.

All these findings are intuitively clear. If a homoskedastic $\mathrm{AR}(1)$ process is contaminated with a dominant white noise process, the OLS estimator is biased towards that white noise process. Consequently, the regression residuals will be autocorrelated, as will be the squared regression residuals. Therefore, the ARCH test will reject the null hypothesis too often compared to the nominal level. If the probability of outliers $\pi$ increases further and $\zeta$ is large, the white noise contamination becomes completely dominant and the regression residuals are approximately equal to the contamination white noise process.

\subsection{Homoskedastic white noise and patchy additive outliers}

Additive outliers can occur either in isolation or in patches. In this subsection, we show that the occurence of only a few adjacent AO's may result in spurious detection of ARCH effects. The effect of patchy additive outliers on the level of the test is thus similar to that of isolated outliers, compare subsection 3.1.

We study the effect of additive outliers that occur in patches of length $k$ on a model that contains neither AR nor GARCH behavior, i.e., $\phi=\tilde{\phi}=\alpha=0$. A patch of outliers occurs if we allow the contamination process $\delta_{t}$ in (9) to be autocorrelated,

$$
\delta_{t}= \begin{cases}\tilde{\delta}_{t} & \text { if } v_{i} \neq 0 \text { for some } i=t-k+1, \ldots, t \\ 0 & \text { else }\end{cases}
$$

with $\tilde{\delta}_{t}$ and $v_{t}$ i.i.d., $P\left(\tilde{\delta}_{t}=1\right)=P\left(\tilde{\delta}_{t}=-1\right)=1 / 2, P\left(v_{t}=0\right)=1-\pi$, and $P\left(v_{t} \neq 0\right)=\pi$. 
As there is no AR parameter to be estimated in the present setting, we directly proceed with the effect of patchy outliers on the estimate of the variance of the regression errors, $\tilde{\sigma}^{2}$. We obtain

$$
\begin{aligned}
\tilde{\sigma}^{2} & =E\left(x_{t}^{2}\right) \\
& =E\left(y_{t}^{2}\right)+\zeta^{2} E\left(\delta_{t}^{2}\right) \\
& =\omega+\zeta^{2}\left(1-P\left(v_{t-k+1}=0, \ldots, v_{t}=0\right)\right) \\
& =\omega+\zeta^{2}\left(1-(1-\pi)^{k}\right)
\end{aligned}
$$

Furthermore, we obtain that

$$
\begin{aligned}
E\left(x_{t}^{2} x_{t-1}^{2}\right)= & E\left(\left(y_{t}+\zeta \delta_{t}\right)^{2}\left(y_{t-1}+\zeta \delta_{t-1}\right)^{2}\right) \\
= & E\left(y_{t}^{2} y_{t-1}^{2}+y_{t}^{2} \zeta^{2} \delta_{t-1}^{2}+y_{t-1}^{2} \zeta^{2} \delta_{t}^{2}+\zeta^{4} \delta_{t}^{2} \delta_{t-1}^{2}\right) \\
= & \omega^{2}+2 \omega \zeta^{2}\left(1-(1-\pi)^{k}\right)+\zeta^{4}\left(P\left(\exists_{i \in\{t-k+1, \ldots, t-1\}}: v_{i} \neq 0\right)+\right. \\
& \left.P\left(v_{t} \neq 0, v_{t-1}=0, \ldots, v_{t-k+1}=0, v_{t-k} \neq 0\right)\right) \\
= & \omega^{2}+2 \omega \zeta^{2}\left(1-(1-\pi)^{k}\right)+\zeta^{4}\left(1-(1-\pi)^{k-1}+(1-\pi)^{k-1} \pi^{2}\right) \\
= & \omega^{2}+2 \omega \zeta^{2}\left(1-(1-\pi)^{k}\right)+\zeta^{4}\left(1-(1-\pi)^{k}(1+\pi)\right) .
\end{aligned}
$$

As a result, the expectation of (10) can be written as

$$
\frac{E\left(x_{t}^{2} x_{t-1}^{2}\right)-\tilde{\sigma}^{4}}{\tilde{\sigma}^{2}}=\frac{\zeta^{4}(1-\pi)^{k+1}\left(1-(1-\pi)^{k-1}\right)}{\omega+\zeta^{2}\left(1-(1-\pi)^{k}\right)} .
$$

Expression (17) clearly demonstrates that unless there are no outliers, i.e., $\zeta=0$ or $\pi=0$, or only outliers, i.e., $\pi=1$, the noncentrality parameter of the ARCH test is nonzero. This results in a rejection frequency of the test above the nominal level, despite the absence of ARCH effects. So AO's occurring in patches can result in a spurious detection of $\mathrm{ARCH}$ effects. This is intuitively clear, as additive outliers result in large values of the innovations. If several of such values occur in a row, the ARCH test is biased towards the detection of volatility clustering, i.e., large innovations following large innovations. If patches become very long $(k \rightarrow \infty)$ or if 
the probability of a patch of outliers occurring is large $(\pi \uparrow 1)$, then the noncentrality parameter tends to zero again. So long patches of dominant patches result in a distribution of the $\mathrm{ARCH}$ test close to its null distribution. Put differently, long patches lead to small size distortions. It can be shown, however, that the same phenomenon for the noncentrality parameter holds under the alternative of genuine ARCH effects, such that long patches of outliers lead to a power loss of the ARCH test. This is again intuitively clear, because in such cases the homoskedastic white noise contamination will dominate the original ARCH signal, such that the volatility clustering will go unnoticed. To get some intuition, one can consider the extreme case of an infinitely long patch of dominant outliers, $k \rightarrow \infty$ and $\zeta \rightarrow \infty$. In that case one no longer observes the original process, but only the contaminating white noise.

\subsection{White noise, $\operatorname{ARCH}(1)$, and isolated additive outliers}

The final effect of additive outliers we demonstrate in this paper concerns the power of the ARCH test. Consider the same model for $y_{t}$ as in subsection 3.2, only with $\varepsilon_{t}$ being $\mathrm{ARCH}(1)$ instead of homoskedastic. The outlier process $\delta_{t}$ is now assumed to be i.i.d. as in subsection 3.1. We first compute the variance of $x_{t}$,

$$
\begin{aligned}
\tilde{\sigma}^{2} & =E\left(x_{t}^{2}\right) \\
& =E\left(y_{t}^{2}\right)+\zeta^{2} E\left(\delta_{t}^{2}\right) \\
& =\omega /(1-\alpha)+\zeta^{2} \pi .
\end{aligned}
$$

Moreover,

$$
\begin{aligned}
E\left(x_{t}^{2} x_{t-1}^{2}\right)= & E\left(\left(y_{t}+\zeta \delta_{t}\right)^{2}\left(y_{t-1}+\zeta \delta_{t-1}\right)^{2}\right) \\
= & \pi^{2} \zeta^{4}+2 \pi \zeta^{2} \omega /(1-\alpha)+E\left(\left(\omega+\alpha y_{t-1}^{2}\right) y_{t-1}^{2}\right) \\
= & \pi^{2} \zeta^{4}+\left(2 \pi \zeta^{2}+\omega\right) \omega /(1-\alpha)+ \\
& 3 \alpha \omega^{2}(1+\alpha) /\left(\left(1-3 \alpha^{2}\right)(1-\alpha)\right),
\end{aligned}
$$


where we assume that $3 \alpha^{2}<1$, such that the unconditional fourth moment of $y_{t}$ exists. As a result, the expectation of (10) can be written as

$$
\frac{E\left(x_{t}^{2} x_{t-1}^{2}\right)-\tilde{\sigma}^{4}}{\tilde{\sigma}^{2}}=\frac{3 \alpha \omega^{2}\left(\alpha^{2}+\alpha+2 / 3\right)}{\left(1-3 \alpha^{2}\right)(1-\alpha)\left(\omega+\zeta^{2} \pi(1-\alpha)\right)} .
$$

Expression (20) clearly demonstrates that the noncentrality parameter is decreasing in both $\pi$ and $\zeta$. As the noncentrality parameter involves the square of (17), we can see that the power of the test decreases very rapidly if the magnitude of the outliers $\zeta$ increases. The intuition behind these results is that, if we have a GARCH process which is contaminated with a dominant homoskedastic white noise process, the test will have difficulty in spotting the GARCH behavior of the underlying uncontaminated series. So, under the alternative, large fractions of contamination or contamination with large outliers both lead to a severe power loss in the present context. In section 6 we will give an empirical example of this phenomenon.

In section 5 we use Monte Carlo simulations to examine if and how the asymptotic results presented in this section carry over to small samples. First however, in the next section we put forward an outlier robust variant of the LM test for ARCH.

\section{An outlier robust test for ARCH}

The results in the previous section show that the LM test for ARCH can be severely distorted by additive outliers. Van Dijk et al. (1996) investigate a similar problem when testing for linearity of the conditional mean. They show that an outlier robust test statistic is obtained if the model under the null hypothesis is estimated by an outlier robust estimator. In particular, they suggest to use a high breakdown point generalized maximum likelihood type (HBP-GM) estimator to have maximum protection against AO's. General introductions to outlier robust estimation techniques can be found in, e.g., Huber (1981), Martin (1981), Hampel et al. (1986), and, more recently, Lucas (1996). The idea of estimating the model under the null using an outlier robust estimator can also be used to robustify the LM test for ARCH in (5). 
For technical details we refer to Van Dijk et al. (1996), here we only present the general concepts involved.

The class of GM estimators can be interpreted as a type of weighted least squares estimator, with the weights chosen endogenously in such a way that influential observations, such as AO's, do not affect the parameter estimates. In particular, in the $\mathrm{AR}(1)$ example the GM estimator solves the first order condition

$$
\sum_{t=1}^{T} y_{t-1} \cdot w_{r}\left(r_{t}\right)\left(y_{t}-\phi y_{t-1}\right)=0
$$

where $w_{r}(\cdot)$ is a weight function, which determines the weight for the $t$-th observation, $r_{t}$ is the standardized residual, $r_{t}=\left(y_{t}-\phi y_{t-1}\right) /\left(\sigma_{\varepsilon} w_{y}\left(y_{t-1}\right)\right)$, with $w_{y}(\cdot)$ a weight function for the regressor $y_{t-1}$, and $\sigma_{\varepsilon}$ is an estimate of the scale of $\varepsilon_{t}$. Both weight functions $w_{r}(\cdot)$ and $w_{y}(\cdot)$ are bounded by zero and one. The first order condition (21) is nonlinear in $\phi$ and, therefore, estimation requires an iterative procedure. In order to have maximum protection against outliers, the breakdown point of the estimator, that is, the maximum fraction of contaminated observations the estimator can cope with, should be as high as possible. Simpson et al. (1992) and Coakley and Hettmansperger (1993) show that if a high breakdown point (HBP) estimator is used to construct starting values for $\phi$ and $\sigma_{\varepsilon}$ and if only one iteration of the weighted least squares scheme is performed, an efficient estimator is obtained which retains the high breakdown point of the initial estimator. The least median of squares (LMS) estimator of Rousseeuw (1984) is used to obtain a starting value for the autoregressive parameter, while the median absolute deviation (MAD) estimator provides an initial scale estimate, i.e., $\hat{\sigma}=1.483 \cdot \operatorname{med}\left(\left|y_{t-1}-\operatorname{med}\left(y_{t-1}\right)\right|\right)$ where med denotes the median. The constant 1.483 is used to make the MAD a consistent estimator of the standard deviation in case $\varepsilon_{t}$ is normally distributed.

Defining $\psi\left(r_{t}\right)=r_{t} w_{r}\left(r_{t}\right)$, we use the polynomial $\psi$ function as proposed in Lucas et al. (1996), given by

$$
\psi\left(r_{t}\right)=r_{t}\left(1-H\left(\left|r_{t}\right|-c_{1}\right)\right) \operatorname{sgn}\left(r_{t}\right)+H\left(\left|r_{t}\right|-c_{1}\right)\left(1-H\left(\left|r_{t}\right|-c_{2}\right)\right) g\left(\left|r_{t}\right|\right),
$$


where $c_{1}$ and $c_{2}$ are tuning constants, $H(\cdot)$ is the Heaviside function, defined by $H(z)=1$ if $z>0$ and $H(z)=0$ otherwise, sgn is the signum function, and $g\left(\left|r_{t}\right|\right)$ is a fifth order polynomial such that $\psi(\cdot)$ is twice continuously differentiable. For this choice of $\psi(\cdot)$, the resulting weight function $w_{r}(\cdot)$ is such that the observation at time $t$ receives a weight equal to 1 if its standardized residual is within $\left(-c_{1}, c_{1}\right)$ and a weight equal to zero if $r_{t}$ is larger than $c_{2}$ in absolute value. Partial weighting occurs in-between. The tuning constants $c_{1}$ and $c_{2}$ are taken to be the square roots of the 0.99 and 0.999 quantiles of the $\chi^{2}(1)$ distribution, that is, $c_{1}=2.576$ and $c_{2}=3.291$.

The weight function $w_{y}(\cdot)$ for the regressor is specified as

$$
w_{y}\left(y_{t-1}\right)=\psi\left(d\left(y_{t-1}\right)^{\alpha}\right) / d\left(y_{t-1}\right)^{\alpha}
$$

where again $\psi(\cdot)$ is given by $(22), d\left(y_{t-1}\right)$ is the Mahalanobis distance of $y_{t-1}$, i.e., $d\left(y_{t-1}\right)=\left|y_{t-1}-m_{y}\right| / \sigma_{y}$, with $m_{y}$ and $\sigma_{y}$ measures of location and scale of $y_{t-1}$, respectively. These measures are estimated robustly by the median and the MAD, respectively. Finally, following Simpson et al. (1992), the constant $\alpha$ in (23) is set equal to 2 in order to obtain robustness of standard errors.

Hampel et al. (1986) and Peracchi (1991) show that the robustness properties of estimators carry over to test statistics based on these estimators. This suggests that the HBP-GM estimator discussed above can be used to construct a robust version of the LM statistic for ARCH. In particular, a robust equivalent to the LM test in (5) is obtained as $T$, the sample size, times the coefficient of determination of a regression of the weighted squared residuals $\psi\left(r_{t}\right)^{2}$ on a constant and $q$ lags. Under conventional assumptions, the outlier robust LM test, which will be denoted by $\xi_{R}$, retains its limiting $\chi^{2}(q)$ distribution. In the next section we compare the small sample properties of $\xi$ and $\xi_{R}$ using Monte Carlo experiments. 


\section{Monte Carlo experiments}

Monte Carlo experiments are conducted to complement the asymptotic results obtained in section 3 by some estimates of the size and power of the standard LM test for $\mathrm{ARCH}$ in small samples in the presence of outliers. In addition, these experiments are used to investigate the properties of the robust test developed in the previous section.

\subsection{Monte Carlo design}

All the models which are used to generate series are nested within the contaminated $\operatorname{AR}(1)-G A R C H(1,1)$ model,

$$
\begin{aligned}
y_{t}-\mu & =\phi\left(y_{t-1}-\mu\right)+\varepsilon_{t}, \quad t=1, \ldots, T, \\
\varepsilon_{t} \mid \Omega_{t-1} & \sim I N\left(0, h_{t}\right) \\
h_{t} & =\omega+\alpha \varepsilon_{t-1}^{2}+\beta h_{t-1}, \\
x_{t} & =y_{t}+\zeta \delta_{t},
\end{aligned}
$$

where $P\left(\delta_{t}=1\right)=P\left(\delta_{t}=-1\right)=\pi / 2$ and $P\left(\delta_{t}=0\right)=1-\pi$. The probability of occurrence of AO's $\pi$ is fixed at 0.05 throughout. In all experiments, $\mu$ is set equal to zero, while $\omega=1-\alpha-\beta$, such that the unconditional variance of the errors $\varepsilon_{t}$ equals 1 for all choices of $\alpha$ and $\beta$. In the Monte Carlo experiments, we study the effects of varying the autoregressive parameter $\phi$, the GARCH-parameters $\alpha$ and $\beta$, the absolute magnitude of the AO's $\zeta$, and the sample size $T$. The necessary starting values for both $y_{t}$ and $\varepsilon_{t}$ are set equal to zero, while the starting value for $h_{t}$ is set equal to the unconditional variance. The first 100 observations of each series are discarded in order to avoid possible dependence of the results on these initial conditions. The AR order is assumed known, while an intercept is always included in the estimation of the linear model under the null hypothesis. 


\subsection{Size}

The effects of isolated outliers on the size of the LM test in small samples are investigated by generating 1000 series according to an AR(1) model with conditionally homoskedastic errors, i.e., (24)-(27) with $\alpha$ and $\beta$ fixed at zero. We set $\phi=0.0,0.1,0.3, \ldots, 0.9, \zeta=0,3,4,5$, and $T=100,250,500$, giving a total of 72 experiments.

\section{- insert Table 1 -}

Rejection frequencies of the standard and robust LM test against $\operatorname{GARCH}(1,1)$ errors using 5\% asymptotic critical values are given in Table 1 (results for tests against higher order ARCH alternatives and other contamination fractions are available on request from the corresponding author). The size of the standard test in case of no outliers corroborates the findings of other Monte Carlo studies mentioned in Section 2 in that the empirical size is below the nominal size. The size of the robust test is quite reasonable as well. In the presence of outliers, the rejection frequencies remain fairly low for small values of the autoregressive parameter for all sample sizes considered. For $\phi=0.7$ or 0.9 however, it is seen that AO's have quite a dramatic effect on the behavior of the standard test, which now rejects the null much more often than expected. This finding confirms the asymptotic result obtained in section 3.1 that the noncentrality parameter of the $\mathrm{ARCH}$ test is larger for larger values of the autoregressive parameter.

The robust test is affected by the presence of AO's as well, albeit to a much lesser extent. Rejection frequencies typically remain below $10 \%$ and $25 \%$ for $T=100$ and 250, respectively. The effects of the magnitude of the outliers on the size estimates for the standard test is seen to accord with the results of section 3.1 as well. Increasing the absolute magnitude of the outliers initially leads to more frequent rejection of the null. For $\zeta=5$ and $T=250$ and 500, it becomes noticeable that the distribution of the LM test approaches the nominal distribution again. By contrast, the rejection 
frequencies for the robust test decline when moving from $\zeta=3$ to $\zeta=4$ as well.

Obviously, a larger outlier is more easily detected by the robust estimation procedure and, therefore, has less effect on the level of the robust test.

\subsection{Power}

The effects on the power of the LM test are investigated by generating 1000 series according to (24)-(27) with $\phi$ fixed at zero (which is assumed known). We set $\alpha=0.1,0.3,0.5,0.7,0.9, \beta=0.2,0.4,0.6,0.8, \zeta=0,3,4,5$, and $T=100,250,500$. Only combinations of the GARCH parameters for which $\alpha+\beta<1$ are considered, leaving a total of 120 experiments.

\section{- insert Table 2 -}

Table 2 shows the rejection percentages for these experiments. The entries in the columns for which $\zeta=0$ indicate that the LM test is more sensitive in the $\alpha$ - than in the $\beta$-direction. The power increases quite rapidly when $\alpha$ gets larger, while it is hardly affected when $\beta$ gets larger. As Lee and King (1993) argue, this is not surprising since the LM test is derived as a test for $\mathrm{ARCH}(1)$ disturbances. Comparing the standard and robust tests, it is seen that in the absence of outliers, there is a power loss when using the robust test compared to the nonrobust test. The loss of power becomes less for larger sample sizes, but remains considerable for combinations of small $\alpha$ and large $\beta$, which are values for the GARCH parameters typically encountered in practice. This power loss has to be considered as a kind of "insurance premium" one has to pay in order to be protected against the bad effects of outliers.

In the presence of outliers, the power of the conventional test drops quite dramatically, confirming the asymptotic result of section 3.3. By contrast, the power of the robust test is hardly affected and is considerably higher than the power of the standard test. Moreover, the power of the robust test is insensitive to the magni- 
tude of the outliers, whereas the OLS based test suffers relatively more from larger outliers.

\section{$6 \quad$ Empirical illustrations}

In this section we present two empirical applications to illustrate the behavior of the LM test for ARCH in the presence of AO's. In Section 6.1, we apply the test as a diagnostic check to the residuals from a model for the quarterly French industrial production index. This example shows that AO's may give rise to spurious indication of the presence of ARCH effects. In Section 6.2, the test is applied to weekly exchange rate returns of the Spanish peseta versus the US dollar. Surprisingly, the standard test does not find any evidence for conditional heteroskedasticity. Application of the robust test to the same series reveals that ARCH effects are masked by only a few outlying observations.

\subsection{ARCH effects in French industrial production?}

In this subsection we illustrate the use of the LM test as diagnostic check to test the adequacy of a fitted model. We consider modelling the quarterly, seasonally unadjusted index of industrial production for France, for which we may expect an AO to appear in the second quarter of 1968 because of the nationwide strike in May of that year. The data cover the period 1960(i)-1987(iv) and are constructed by averaging the corresponding monthly observations, which are taken from the $O E C D$ Main Economic Indicators. The data are made approximately stationary by taking seasonal differences of the logarithms. The resulting series is graphed in the left panel of Figure 2. Two marked features of this series stand out from this graph. First, the 1975 recession following the first oil crisis is clearly visible, leading to five subsequent quarters of negative growth. Second, the observations 1968(ii) and 1969(ii) show a pattern which is typical for an additive outlier in the level of the series, i.e., a large dip for the first observation and a strong peak for the second one. 


\section{- insert Figure 2 -}

A specification search leads to an AR model for this series with lags 1, 4, 5, 8 and 9 included as regressors. The standard LM test for $\operatorname{ARCH}(q)$ is applied to the residuals from this model, where $q$ ranges from 1 to 12 . The outcomes of the tests are set out in the second column of Table 3. The $p$-values of the tests do not exceed conventional significance levels, and hence, based on this standard test, we would conclude there is very strong evidence for the presence of $\mathrm{ARCH}$.

\section{- insert Table 3 -}

To check the robustness of this result to the possible presence of AO's, we apply the robust LM test for ARCH. The weights assigned by estimating the selected AR model using our robust method are displayed in the right part of Figure 2. It is seen that 18 observations (out of a total of 99) are downweighted, while 15 of these observations receive a weight equal to zero. The corresponding observations of the quarterly difference series are marked with circles in the left panel of the same figure. Most of these observations are associated with either the 1968 strike or the 1975 recession. Note that this relatively large number of zero weights does not imply that the series contains a large number of outliers, but merely that the AO's affect many subsequent observations, due to the large AR structure. The robust test results along with the corresponding $p$-values, given in the third column of Table 3 clearly demonstrate that all evidence for the presence of ARCH disappears. Hence, we conclude that the previous suggestion of heteroskedasticity in French industrial production is caused by aberrant observations associated with the strike in May 1968 and the oil crisis around 1974.

The LM test for ARCH has power against a range of other alternatives as well. This can be most easily understood by noting that it is asymptotically equivalent to the portmanteau test of McLeod and Li (1983). Hence, one might argue that it is somewhat naive to take the results of the standard LM test as equivalent to the 
presence of $\mathrm{ARCH}$ in the French industrial production series, and that they rather should be interpreted as a sign of general misspecification of the conditional mean. Perhaps it is good to remark that the robust test is useful in this respect as well, because the weights obtained from the robust estimation procedure indicate which observations cause the model to be misspecified.

\subsection{ARCH effects in the Spanish peseta exchange rate!}

The GARCH $(1,1)$ model has been frequently applied to (high frequency) financial time series such as exchange rates. Examples include Baillie and Bollerslev (1989) and Hsieh (1989). In this section, we apply the standard and robust LM tests for ARCH to weekly returns for the Spanish peseta against the US dollar. The data are obtained from the database of the Federal Reserve Bank of Chicago and consist of Wednesday noon bid rates from January 1, 1986 until December 27, 1995. In case the market was closed on a particular Wednesday, the observation on the following Thursday is used. The return series was created by taking first differences of the logarithm of the exchange rate and is graphed in the left panel of Figure 3.

\section{- insert Figure 3 - \\ - insert Table 4 -}

The upper panel of Table 4 gives some summary statistics for the return series. The median is seen to differ substantially from the mean, while the skewness is significantly positive as well, suggesting asymmetry in this series. The value of the kurtosis is typical for many financial time series, being substantially above the normal value of 3 . By contrast, the autocorrelation coefficients of the centered and squared returns, given in the middle panel of Table 4, do not fit the pattern characteristic for financial time series of being small but significant for a large number of lags. In fact, of the first twenty autocorrelations, only the third and twentieth are significantly different from zero. This may be regarded as a first suggestion of 
additive outliers, since isolated AO's bias the low order autocorrelation coefficients towards zero. This conjecture is strengthened by the observation that the autocorrelations at some extremely high lags such as 35, 43 and 78 (not reported here) suddenly become significant again.

Following Baillie and Bollerslev (1989), we assume that exchange rate returns are uncorrelated, possible with nonzero mean $\mu$. Testing for $\mathrm{ARCH}$ in the residuals can now be done by simply applying the LM test against $\operatorname{ARCH}(q)$ to the demeaned and squared return series. The test results for $q$ ranging from 1 until 13 are set out in the second column of the bottom panel of Table 4 . The $p$-values for the standard test are well above conventional significance levels, giving no rise to reject the null hypothesis. This result is rather puzzling, even more so because estimating a GARCH(1,1) model for this series gives significant estimates for the parameters in the conditional variance equation, i.e., $\alpha=0.122$ and $\beta=0.676$ with standard errors equal to 0.047 and 0.097 , respectively.

We apply the robust test to check whether the standard test results might be driven by neglecting the presence of AO's. The drift is now estimated as the median of the return series. The $p$-values associated with the robust test, shown in the third column of the bottom panel of Table 4, indicate that ARCH effects are present indeed. Only for $q=1$ there may be some doubt, as the test rejects the null only at the $14.4 \%$ significance level. Inspection of the weights from the robust estimation procedure, shown in the right panel of Figure 3, reveals that from the total 521 observations, only 13 are are downweighted. Notice that nine of these observations do not receive a weight equal to zero. Hence, our robust estimation method allow for a less harsh treatment of aberrant observations. As can be seen from Figure 3, the downweighted observations concern extreme or outlying values of absolute returns. Whether these observations can be modeled by the AO model depends on the events that have taken place on these specific dates. If no particular events can be identified, the modeler might conclude that a leptokurtic conditional distribution 
is needed for the present exchange rate return series.

\section{Concluding remarks}

In this paper we proposed a robust LM test for conditional heteroscedasticity. The need for such a robust test is motivated by both analytical derivations and Monte Carlo simulations, which show that the standard LM test of Engle (1982) breaks down in the presence of outliers. The robust test uses a HBP-GM estimator to estimate the homoskedastic model under the null hypothesis. The Monte Carlo evidence suggests that the resulting test offers much better protection against the influence of outliers than the standard test. The application to weekly exchange rate returns shows that hidden ARCH can be revealed by taking care of outliers. Furthermore, the French industrial production example shows that obviously neglected AO's suggest spurious ARCH.

Further research should include a comparison of our robust test with the robust test of Bollerslev and Wooldridge (1992). Furthermore, we aim to investigate the estimation of GARCH models using robust methods as well as the forecasting properties of robustified GARCH models for out-of-sample volatility. The tentative

results in Franses and Ghijsels (1995) seem to indicate that quite some forecasting power can be gained. Finally, it is interesting to study how our robust test performs for other financial time series, possibly sampled at different frequencies, such as days or minutes. 


\section{References}

Baillie, R.T. and T. Bollerslev (1989) The message in daily exchange rates: a conditional variance tale, Journal of Business and Economic Statistics, 7, 297-305.

Bera, A.K. and M.L. Higgins (1993) A survey of ARCH models: properties, estimation and testing, Journal of Economic Surveys, 7, 305-366.

Bollerslev, T. (1986) Generalized autoregressive conditional heteroscedasticity, Journal of Econometrics, 31, 307-327.

Bollerslev, T. and J.M. Wooldridge (1992) Quasi-maximum likelihood estimation and inference in dynamic models with time-varying covariances, Econometric Reviews, 11, 143-172.

Bollerslev, T., R.F. Engle and D.B. Nelson (1994) ARCH models, in R.F. Engle and D.L. McFadden, eds., Handbook of Econometrics IV, 2961-3038, Amsterdam, NorthHolland.

Bollerslev, T., R.Y. Chou and K.F. Kroner (1992) ARCH modeling in finance: a review of the theory and empirical evidence, Journal of Econometrics, 52, 5-59.

Breusch, T.S. and A.R. Pagan (1979) A simple test for heteroscedasticity and random coefficient variation, Econometrica, 47, 1287-1294.

Bustos, O.H. and V.J. Yohai (1986) Robust estimates for ARMA models, Journal of the American Statistical Association, 81, 155-168.

Coakley, C.W. and T.P. Hettmansperger (1993) A bounded influence, high breakdown, efficient regression estimator, Journal of the American Statistical Association, 88, $872-880$.

Denby, L. and R.D. Martin (1979) Robust estimation of the first-order autoregressive parameter, Journal of the American Statistical Association, 74, 140-146.

Diebold, F.X. and P. Pauly (1989) Small sample properties of asymptotically equivalent tests for autoregressive conditional heteroscedasticity, Statistical Papers, 30, 105-131.

Engle, R.F. (1982) Autoregressive conditional heteroscedasticity with estimates of the variance of United Kingdom inflation, Econometrica, 50, 987-1007.

Franses, P.H. and H. Ghijsels (1995) Additive outliers, GARCH and forecasting volatility, Manuscript under revision for publication, Econometric Institute, Erasmus University Rotterdam.

Gregory, A.W. (1989) A nonparametric test for autoregressive conditional heteroscedasticity: a Markov-chain approach, Journal of Business and Economic Statistics, 7, $107-115$.

Hampel, H.R., E.M. Ronchetti, P.J. Rousseeuw and W.A. Stahel (1986) Robust Statistics - The Approach based on Influence Functions, John Wiley \& Sons, New York.

Hsieh, D.A. (1989) Modeling heteroscedasticity in daily foreign-exchange rates, Journal of Business and Economic Statistics, 7, 307-317. 
Huber, P.J. (1981) Robust Statistics, Wiley, New York.

Koenker, R. (1981) A note on studentizing a test for heteroscedasticity, Journal of Econometrics, 17, 107-112.

Lee, J.H.H. (1991) A Lagrange multiplier test for GARCH models, Economics Letters, 37, 265-271.

Lee, J.H.H. and M.L. King (1993) A locally most mean powerful based score test for ARCH and GARCH regression disturbances, Journal of Business and Economic Statistics, $11,17-27$.

Lucas, A. (1996) Outlier Robust Unit Root Testing, PhD thesis, Thesis/Tinbergen Institute, Amsterdam.

Lucas, A., R. van Dijk and T. Kloek (1996) Outlier robust GMM estimation of leverage determinants in linear dynamic panel data models, Tinbergen Institute Discussion paper 94-132.

Martin, R.D. (1981) Robust methods for time series, in D.F. Findley, ed., Applied Time Series Analysis, 6843-759, Academic Press, New York.

Martin, R.D. and V.J. Yohai (1986) Influence functionals for time series, Annals of Statistics, 14, 781-818.

McLeod, A.I. and W.K. Li (1983) Diagnostic checking ARMA time series models using squared-residual autocorrelations, Journal of Time Series Analysis, 4, 269-273.

Peracchi, F. (1991) Robust M-tests, Econometric Theory, 7, 69-84.

Rousseeuw, P.J. (1984) Least median of squares regression, Journal of the American Statistical Association, 79, 871-880.

Simpson, D.G., D. Ruppert and R.J. Carroll (1992) On one-step GM estimates and stability of inferences in linear regression, Journal of the American Statistical Association, 87, 439-450.

Teräsvirta, T. (1996) Two stylized facts and the GARCH(1,1) model, Working paper no. 96, Stockholm School of Economics.

van Dijk, D., P.H. Franses and A. Lucas (1996) Testing for smooth transition nonlinearity in the presence of additive outliers, Econometric Institute Report 9622/A, Erasmus University Rotterdam.

Weiss, A.A. (1986) Asymptotic theory for ARCH models: estimation and testing, Econometric Theory, 2, 107-131. 
Table 1: Size of LM test for $\mathrm{ARCH}(1)$ disturbances (5\% outliers)

\begin{tabular}{|c|c|c|c|c|c|c|c|c|c|c|}
\hline & \multirow[b]{2}{*}{$\phi$} & \multirow[b]{2}{*}{$\zeta$} & \multicolumn{4}{|c|}{$\overline{\xi \xi}$} & \multicolumn{4}{|c|}{$\xi_{R}$} \\
\hline & & & 0 & 3 & 4 & 5 & 0 & 3 & 4 & 5 \\
\hline \multirow[t]{6}{*}{$T=100$} & 0.0 & & 3.8 & 2.7 & 2.5 & 2.5 & 4.7 & 4.1 & 3.7 & 4.7 \\
\hline & 0.1 & & 4.0 & 1.8 & 2.0 & 2.6 & 4.6 & 4.0 & 4.5 & 4.0 \\
\hline & 0.3 & & 3.2 & 4.0 & 3.7 & 3.7 & 3.3 & 5.8 & 6.4 & 5.1 \\
\hline & 0.5 & & 4.0 & 7.9 & 8.4 & 6.8 & 5.1 & 7.6 & 8.7 & 8.3 \\
\hline & 0.7 & & 2.8 & 14.7 & 17.6 & 15.5 & 2.8 & 9.3 & 10.0 & 6.3 \\
\hline & 0.9 & & 3.8 & 42.1 & 60.0 & 60.9 & 4.4 & 14.7 & 10.1 & 7.1 \\
\hline \multirow[t]{6}{*}{$T=250$} & 0.0 & & 3.0 & 2.6 & 2.4 & 2.3 & 3.0 & 4.7 & 5.2 & 5.1 \\
\hline & 0.1 & & 2.7 & 2.9 & 3.0 & 2.8 & 2.7 & 4.6 & 5.3 & 6.1 \\
\hline & 0.3 & & 3.9 & 6.3 & 5.7 & 5.6 & 3.9 & 7.4 & 6.1 & 6.6 \\
\hline & 0.5 & & 5.0 & 12.5 & 11.4 & 9.2 & 5.0 & 11.6 & 10.8 & 9.6 \\
\hline & 0.7 & & 4.6 & 40.7 & 46.4 & 38.3 & 4.5 & 23.8 & 14.3 & 8.6 \\
\hline & 0.9 & & 3.7 & 85.4 & 96.7 & 96.6 & 3.7 & 25.7 & 7.7 & 4.8 \\
\hline \multirow[t]{6}{*}{$T=500$} & 0.0 & & 4.8 & 4.0 & 4.4 & 4.1 & 5.6 & 6.4 & 8.1 & 8.9 \\
\hline & 0.1 & & 4.2 & 3.8 & 3.5 & 3.3 & 5.2 & 6.7 & 9.9 & 8.1 \\
\hline & 0.3 & & 4.2 & 6.2 & 6.1 & 5.8 & 3.9 & 11.3 & 16.5 & 11.3 \\
\hline & 0.5 & & 3.6 & 24.8 & 23.8 & 16.3 & 4.5 & 20.2 & 26.3 & 14.2 \\
\hline & 0.7 & & 4.4 & 69.6 & 76.5 & 68.4 & 4.0 & 37.5 & 27.7 & 9.0 \\
\hline & 0.9 & & 4.4 & 99.6 & 100.0 & 100.0 & 4.2 & 43.9 & 14.2 & 5.8 \\
\hline
\end{tabular}

Note: Rejection frequencies at $5 \%$ significance level using asymptotic critical values for series generated by (24)-(27) with $\mu=0, \omega=1, \alpha=\beta=0$. Additive outliers are added with probability 0.05 . The table is based on 1000 replications. 
Table 2: Power of LM test for ARCH(1) disturbances (5\% outliers)

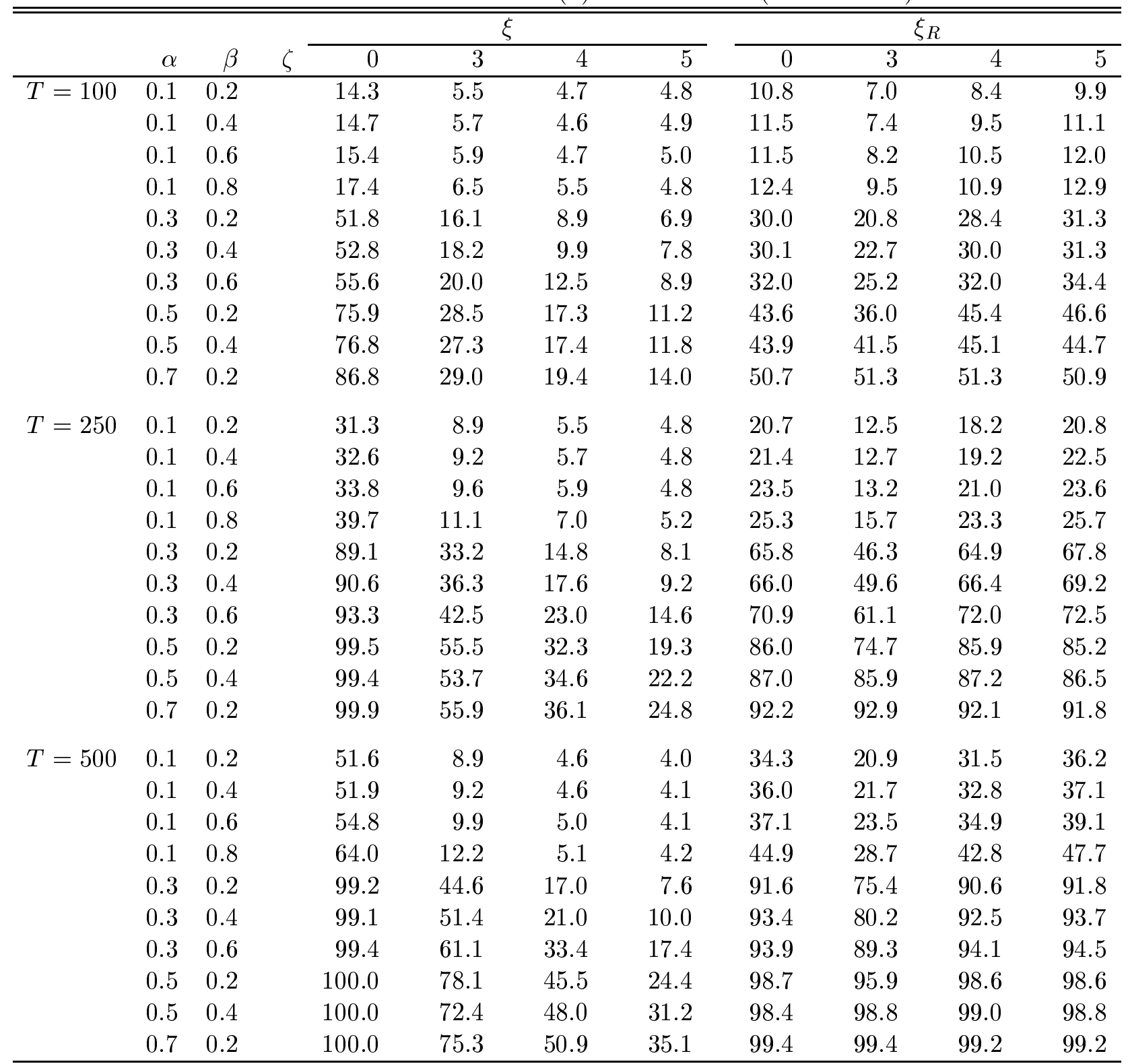

Note: Rejection frequencies at $5 \%$ significance level using asymptotic critical values for series generated by (24)-(27) with $\mu=0, \phi=0, \omega=1-\alpha-\beta$. Additive outliers are added with probability 0.05 . The table is based on 1000 replications. 
Table 3: Tests for ARCH in French industrial production

\begin{tabular}{rcc}
\hline \hline$q$ & $\xi$ & $\xi_{R}$ \\
\hline 1 & $20.172(0.000)$ & $1.226(0.268)$ \\
2 & $27.079(0.000)$ & $3.002(0.223)$ \\
3 & $37.052(0.000)$ & $3.095(0.377)$ \\
4 & $37.525(0.000)$ & $3.538(0.472)$ \\
5 & $37.700(0.000)$ & $6.048(0.302)$ \\
6 & $38.152(0.000)$ & $4.321(0.633)$ \\
7 & $39.074(0.000)$ & $4.696(0.697)$ \\
8 & $39.206(0.000)$ & $4.669(0.792)$ \\
9 & $39.624(0.000)$ & $6.390(0.700)$ \\
10 & $39.593(0.000)$ & $6.961(0.729)$ \\
11 & $39.690(0.000)$ & $6.071(0.869)$ \\
12 & $39.812(0.000)$ & $5.937(0.919)$ \\
\hline
\end{tabular}

Note: Sample period is 1961(i)-1987(iv). The tests are applied to the residuals of an $\operatorname{AR}(1,4,5,8,9)$ model for quarterly differences of the French industrial production index. Asymptotic $p$-values of the LM test are given in parentheses. 
Table 4: Spanish peseta/US dollar exchange rate

\begin{tabular}{lr}
\hline \hline \multicolumn{2}{c}{ Summary statistics } \\
\hline Mean & -0.045 \\
Median & -0.108 \\
Std. dev. & 1.545 \\
Skewness & 0.964 \\
Kurtosis & 7.806 \\
Minimum & -4.423 \\
Maximum & 10.743 \\
\hline
\end{tabular}

Autocorrelation of squared observations

\begin{tabular}{crrr}
\hline \hline Lags & \multicolumn{3}{c}{ Lags } \\
\hline 1 & 0.020 & 11 & 0.015 \\
2 & 0.045 & 12 & 0.003 \\
3 & 0.089 & 13 & 0.019 \\
4 & 0.012 & 14 & 0.008 \\
5 & 0.052 & 15 & 0.003 \\
6 & 0.008 & 16 & 0.011 \\
7 & 0.035 & 17 & -0.006 \\
8 & 0.041 & 18 & 0.003 \\
9 & -0.027 & 19 & -0.007 \\
10 & -0.017 & 20 & 0.087 \\
\hline
\end{tabular}

Note: Standard error of autocorrelations is 0.044 .

LM test for ARCH

\begin{tabular}{rcr}
\hline \hline $\mathrm{q}$ & $\xi$ & \multicolumn{1}{c}{$\xi_{R}$} \\
\hline 1 & $0.195(0.659)$ & $2.131(0.144)$ \\
2 & $1.240(0.538)$ & $9.176(0.010)$ \\
3 & $5.204(0.157)$ & $13.647(0.003)$ \\
4 & $5.253(0.262)$ & $16.572(0.002)$ \\
5 & $6.228(0.285)$ & $16.694(0.005)$ \\
6 & $6.210(0.400)$ & $16.635(0.011)$ \\
7 & $6.719(0.459)$ & $17.520(0.014)$ \\
8 & $7.213(0.514)$ & $17.606(0.024)$ \\
9 & $7.743(0.560)$ & $21.869(0.009)$ \\
10 & $8.132(0.616)$ & $21.847(0.016)$ \\
11 & $8.193(0.696)$ & $22.605(0.020)$ \\
12 & $8.168(0.772)$ & $24.462(0.018)$ \\
13 & $8.356(0.820)$ & $24.659(0.026)$ \\
\hline
\end{tabular}

Note: Sample period is $1 / 8 / 1986-12 / 27 / 1995$. Asymptotic $p$-values of the LM test are given in parentheses. 
Figure 1: Non-centrality parameter of LM test for ARCH in the presence of AO's

$$
\pi=0.01
$$

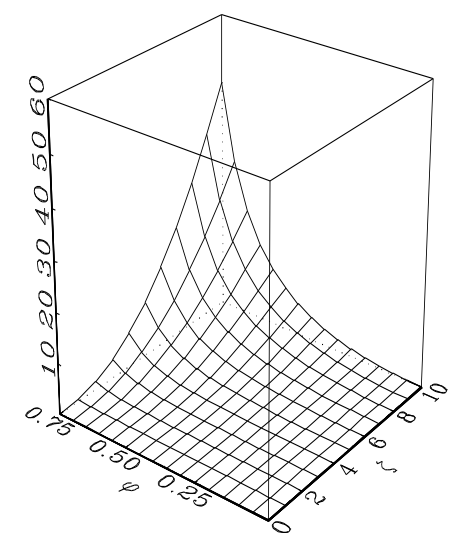

$$
\pi=0.10
$$

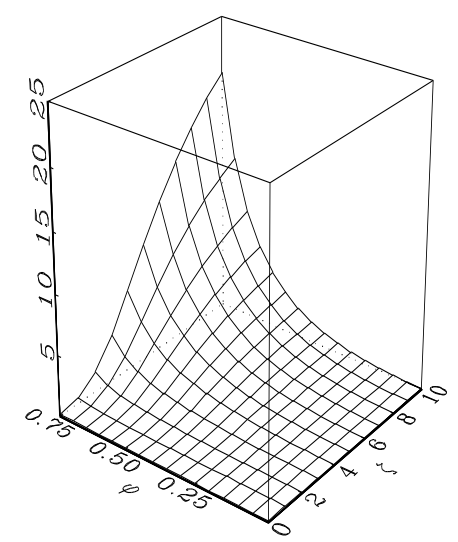

$$
\pi=0.05
$$

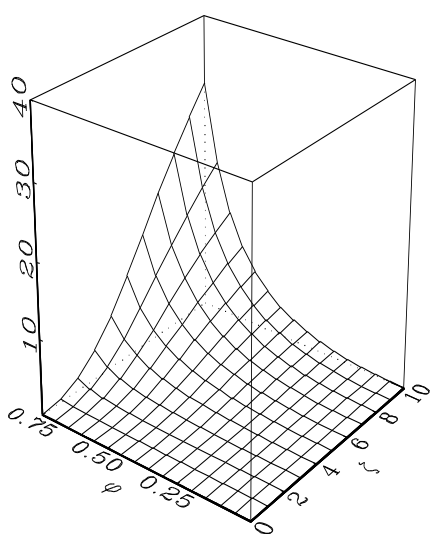

$$
\pi=0.25
$$

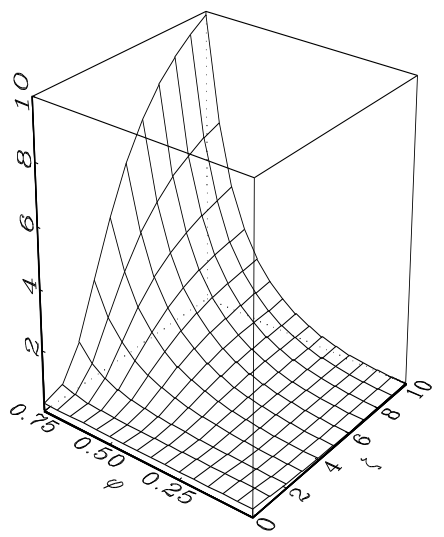

Note: Expected values of the numerator of the noncentrality parameter of the asymptotic distribution of the OLS-based LM test statistic for $\mathrm{ARCH}(1)$ applied to the residuals of an $\mathrm{AR}(1)$ model, given in (10) 
Figure 2: French industrial production: seasonal differences and weights
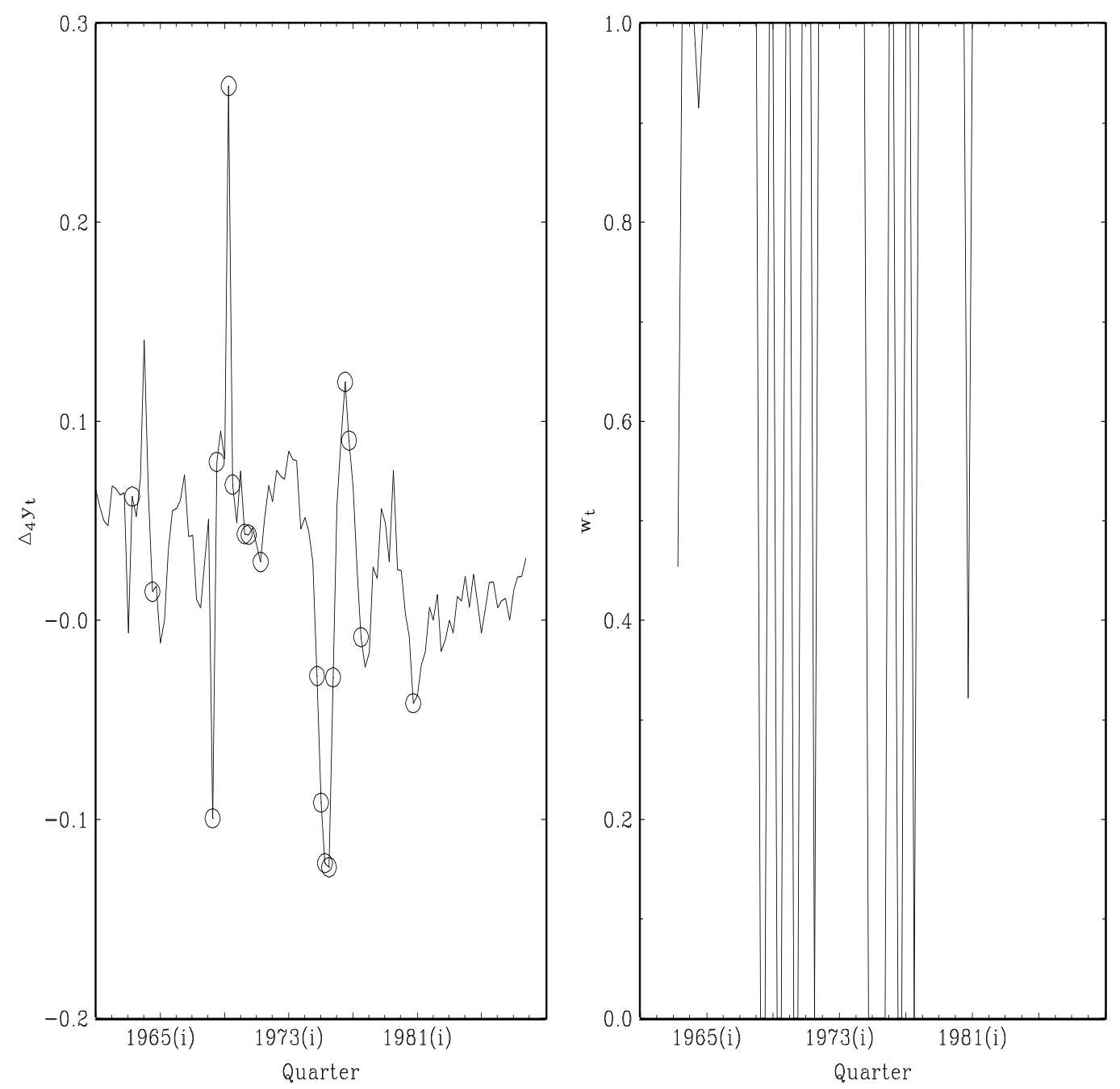

Note: Quarterly differences of French industrial production index, 1961(i)-1987(iv) (left panel) and weights obtained from robust estimation of autoregressive model containing lags 1,4,5,8 and 9 (right panel). 
Figure 3: Spanish peseta/US dollar exchange rate: weekly returns and weights
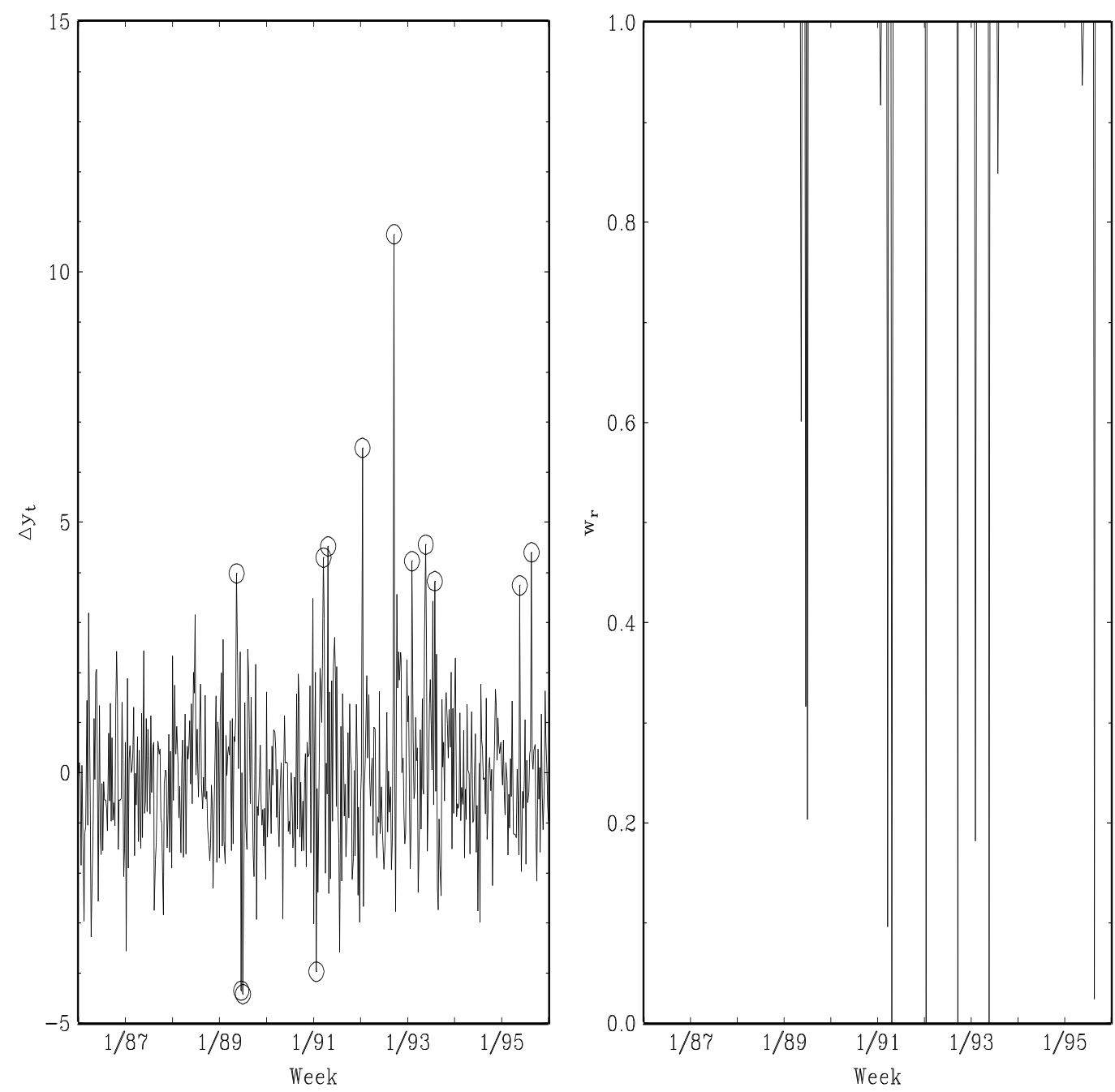

Note: Weekly exchange rate returns for the Spanish peseta versus US dollar, over the period 1/8/1986 until 12/27/1995 (left panel), together with weights assigned by robust estimation of drift term (right panel) 